\title{
Vaccination in rheumatic disease patients: results of a pilot quality improvement program
}

\author{
Gabriel Figueroa-Parra ID , Andrea Moreno-Salinas ID, Leticia Santoyo-Fexas ID, \\ Carmen Magdalena Gamboa-Alonso ID , Juan Pablo Carrizales-Luna ID, \\ Ivan de Jesus Hernandez-Galarza ID , Dionicio Angel Galarza-Delgado ID, \\ Jorge Antonio Esquivel-Valerio ID
}

Rheumatology Service, University Hospital “Dr. José Eleuterio González”, Universidad Autónoma de Nuevo León, Monterrey, México

\begin{abstract}
Objectives: Patients with rheumatic diseases (RD) are at increased risk of infections. Vaccination is recognized as a successful public health measure and is recommended for RD patients. The aim of this study was to describe the strategies implemented in an academic rheumatology outpatient clinic as part of a fellow-in-training-led vaccination quality improvement (QI) program and to explore the vaccination uptake before and one year after the implementation.

Material and methods: The program's objective is the promotion of vaccination among patients and rheumatology fellows (by educational interventions, development of vaccination charts and orders, and modifications to electronic medical records to register vaccination dates and generate reminders). As part of the continuous evaluation of the QI program, a descriptive cross-sectional study was performed to evaluate vaccine uptake pre- and post-interventions and vaccination barriers one year after implementation. Consecutive patients with RD answered a self-administered questionnaire. Results are shown as descriptive statistics. Results: Before the program started 73 patients were surveyed and 102 patients one year after. The vaccination uptake rates for influenza pre- and post-interventions were $43 \%$ and $55 \%$; for pneumococcal vaccination they were $26 \%$ and $30 \%$; for herpes zoster they were $0 \%$ and $4 \%$; for human papillomavirus they were $4 \%$ and $10 \%$; for hepatitis B (HBV) they were $19 \%$ and $25 \%$ respectively. Eighty percent of patients reported some barriers to receiving any of the previous vaccines. The three main reasons for not receiving a vaccine were the lack of recommendation, the lack of availability, and the belief that vaccines do not work. Conclusions: The implementation of a pilot vaccination QI program led by rheumatology fellows-in-training showed promising preliminary benefits in the vaccination uptake among RD patients and helps to evaluate the barriers to surpass.
\end{abstract}

Key words: rheumatic diseases, quality improvement, vaccination, vaccine uptake.

\section{Introduction}

Patients with rheumatic diseases (RD) are at increased risk of infections; this is attributed to the underlying RD, comorbidities, and treatments [1]. Although many infectious diseases can be prevented by vaccines, immunization in this population remains suboptimal, due in part to uncertainty about their efficacy and safety under these clinical situations [2].
Vaccination is recognized as one of the most successful public health measures. Unfortunately, it is perceived as unsafe and unnecessary by a growing number of individuals. Lack of confidence in vaccines is now considered a threat to success in vaccination programs and a public health challenge [3].

Despite advances in recent years, infections remain a major cause of morbidity and mortality in patients 
with RD. Vaccination against preventable illnesses is recommended for patients with $\mathrm{RD}$ by most national and international medical societies [2, 4-8]. Quality improvement $(\mathrm{Q} I)$ interventions are effective in improving vaccination uptake among RD patients $[9,10]$.

The aim of this study was to describe the strategies implemented in a rheumatology outpatient clinic from a university hospital to improve the vaccination knowledge, barriers, and uptake among RD patients. These strategies were part of a pilot fellow-in-training-led QI program. As a secondary aim, we surveyed the vaccination uptake just before the QI program started and one year after their implementation and present these results.

\section{Material and methods}

\section{Design of the quality improvement vaccination program}

The program's objective is the promotion of vaccination among patients and rheumatology fellows. The promotion in patients was done by the distribution and availability of vaccination brochures and an educational community speech (yearly) for this particular population. The promotion among rheumatology fellows was done through the development and distribution of a vaccination manual for rheumatic patients, the development of specific vaccination charts and vaccination passes to be used to send patients to the preventive medicine clinic for vaccination.

We also performed modifications to the electronic medical record to register the vaccine and date of vaccination, and to generate a reminder when it was pertinent. All these strategies were planned by rheumatology fellows-in-training. Figure 1 shows a simplified schematic algorithm of the program.

\section{Vaccination uptake and barriers}

A descriptive cross-sectional study was performed to assess the vaccination uptake in RD patients just be- fore and one year after the pilot implementation of the QI program. The first survey was done one month before starting the program and was repeated in an independent sample in September 2019 (one year after the program).

The implementation of the QI program started in August 2018. During the second survey, besides vaccination uptake, we evaluated the barriers for vaccination expressed by patients after the QI program was running for one year, as part of a continuous evaluation and improvement.

During a structured interview, a self-administered questionnaire was used to obtain demographic characteristics (age, gender, diagnosis). We survey the vaccination status according to the current recommendations for adults, including influenza (last year), pneumococcal (last five years), herpes zoster (ever), human papillomavirus (HPV) (any dose), and hepatitis B (HBV) (any dose) vaccines.

To evaluate vaccination barriers after a year the program was implemented, during the second survey, the question "If you did not receive any of the previous vaccines, what was the reason?" with 6 prespecified options including an open "other reason" option was included in the questionnaire.

\section{Patients and setting}

Two samples of patients were evaluated from our rheumatology outpatient clinic. These patients were derived from each one of the surveys (pre- and post-intervention). We included consecutive patients with a confirmed RD (diagnosed by a fellow-in-training supervised by a certified rheumatologist) who agreed to participate and answer the questionnaire. As an exploratory aim and part of the QI program continuous evaluation, a prespecified sample size was not calculated.

The study was conducted in compliance with the Declaration of Helsinki and the Good Clinical Practice guidelines. Local ethics committee approval was waived due to the descriptive and anonymous nature of the study. Permissions from the research coordination and

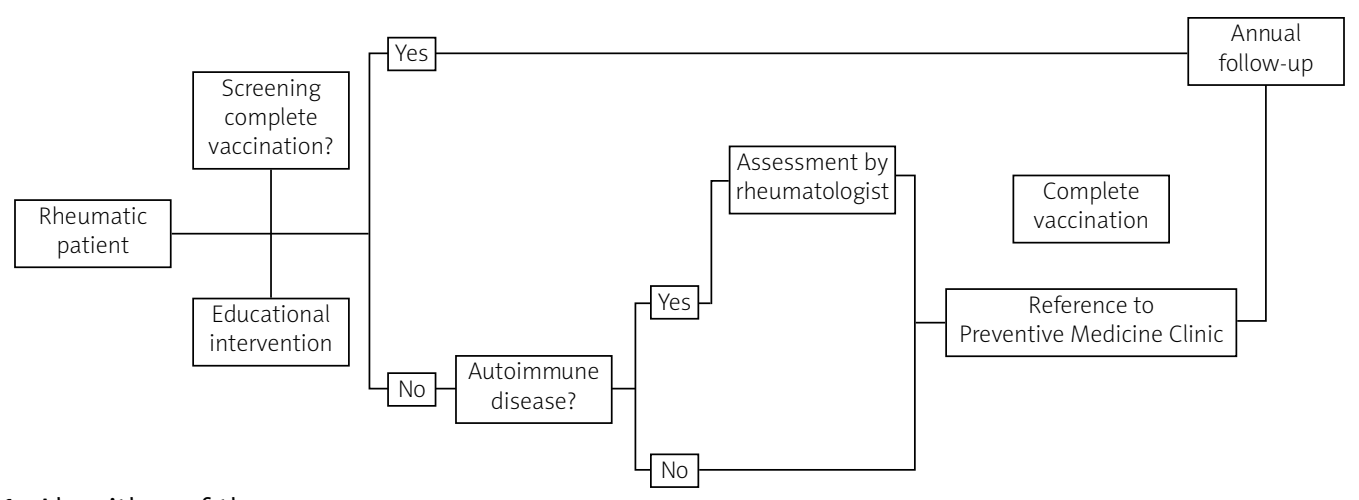

Fig. 1. Algorithm of the program. 
Table I. Demographic characteristics of rheumatic disease patients sampled during the first and second survey. Before and after a year of implementation of a vaccination quality improvement program, respectively

\begin{tabular}{|c|c|c|c|}
\hline $\begin{array}{l}\text { Patients' } \\
\text { characteristics }\end{array}$ & $\begin{array}{c}\text { First } \\
\text { survey } \\
n=73\end{array}$ & $\begin{array}{c}\text { Second } \\
\text { survey } \\
N=102\end{array}$ & $p$-value \\
\hline Age, years [mean \pm SD] & $50.9 \pm 12.4$ & $51.3 \pm 14.7$ & 0.850 \\
\hline Female $[n(\%)]$ & $69(94.5)$ & $84(82.4)$ & 0.017 \\
\hline \multicolumn{4}{|l|}{ Diagnosis [n (\%)] } \\
\hline Rheumatoid arthritis & $48(65.7)$ & $71(69.6)$ & 0.586 \\
\hline $\begin{array}{l}\text { Systemic lupus } \\
\text { erythematosus }\end{array}$ & $7(9.5)$ & $13(12.7)$ & 0.512 \\
\hline Spondyloarthritis & $5(6.8)$ & $2(2.0)$ & 0.111 \\
\hline Other AID & $5(6.8)$ & $4(3.9)$ & 0.391 \\
\hline Non-AID & $8(10.9)$ & $12(11.8)$ & 0.854 \\
\hline
\end{tabular}

AID - autoimmune disease, SD - standard deviation.

Table II. Vaccination barriers identified in rheumatic disease patients after a year of running a quality improvement vaccination program $(N=102)$

\begin{tabular}{|lcc|}
\hline Vaccination barriers & $N(\%)$ & $95 \% \mathrm{Cl}$ \\
\hline $\begin{array}{l}\text { If you did not receive any of the } \\
\text { previous vaccines, what was } \\
\text { the reason? }\end{array}$ & $82(80.4)$ & $71.6-87.0$ \\
\hline It was not recommended & $22(26.8)$ & $18.4-37.5$ \\
\hline Lack of availability & $21(25.6)$ & $17.3-36.1$ \\
\hline Vaccines do not work & $13(15.8)$ & $9.4-25.4$ \\
\hline Fear of adverse events & $8(9.7)$ & $4.8-18.3$ \\
\hline Previous adverse event & $3(3.6)$ & $0.8-10.6$ \\
\hline Other reason & $8(9.7)$ & $4.8-18.3$ \\
\hline Own decision & $7(8.5)$ & $3.9-16.9$ \\
\hline Disinformation & & \\
\hline
\end{tabular}

the head of the rheumatology service were obtained. All the participants were informed of the purpose of the survey, and verbal consent was obtained before their inclusion.

\section{Statistical analysis}

Results are shown as mean and standard deviation (SD) or frequencies and percentages with $95 \% \mathrm{Cl} . \chi^{2}$ or Fisher's exact test was performed to compare proportions before and after the program was implemented. A $p$-value $\leq 0.05$ was considered statistically significant. The analyses were performed using the IBM SPSS Statistics for Windows v.22 statistical package (IBM Corp., Armonk, N.Y., USA).

\section{Results}

Before the program started, 73 patients were surveyed: the mean age was 50.9 (SD 12.4) years, and $94.5 \%$ were women. During the second survey after the program was implemented, 102 patients were included with a mean age of 51.3 (SD 14.7) years, of whom $82.4 \%$ were women. The demographic data and diagnoses are shown in Table I.

The vaccination uptake rates reported pre- and post-interventions for influenza vaccine were $43 \%$ and $55 \%$ ( $p=0.118)$; for pneumococcal vaccine were $26 \%$ and $30 \%(p=0.563)$; for herpes zoster vaccine were $0 \%$ and $4 \%(p=0.084)$; for HPV vaccine were $4 \%$ and $10 \%(p=0.138)$; and for HBV vaccine were $19 \%$ and $25 \%$ $(p=0.350)$ respectively.

Eighty-two (80.4\%) patients reported some barriers to receive any of the aforementioned vaccines. The three main reasons for not receiving a vaccine were the lack of recommendation from their rheumatologist, the lack of availability of vaccines, and the belief that vaccines do not work (Table II).

\section{Discussion}

Vaccination among RD patients is broadly recommended [2, 4-8]. Unfortunately, vaccination remains low in this population [11-15]. Here, we describe costless strategies framed under a QI program led by rheumatology fellows-in-training to improve general vaccination uptake, assess vaccination knowledge, and evaluate barriers in vaccination to be surpassed.

Our results showed a slight but not significant improvement in vaccination uptake of 12\% for influenza, $6 \%$ for HPV and HBV, and 4\% for pneumococcal pneumonia and herpes zoster vaccines after the first year of implementation of our QI program.

These comparisons were not significant, probably due to the small sample size and the fact that this study was not designed to identify this difference. Nevertheless, as preliminary results they could give an idea of the potentially positive impact of the QI program in the vaccination uptake of RD patients.

Other QI interventions consisting of paper reminders to improve pneumococcal vaccination in RD patients have also shown improvement in the prevalence of pneumococcal vaccination [9].

Another multi-level program that included electronic reminders with linked orders, physician and patient interventions also demonstrated improvement in pneumococcal, influenza, and herpes zoster vaccination rates [10]. 
We know that with this slight improvement in the vaccination uptake, we cannot make a definitive conclusion, but as preliminary results of the first year, they show promising benefits that hopefully will accumulate during the following years.

A high proportion of our patients reported barriers to receiving the vaccines evaluated. The main barrier reported was that their physician did not recommend it, followed by the lack of availability, the belief that vaccines do not work, and finally, concerns associated with adverse events. It is reported that most patients state they would get a vaccine on the advice of their physician, and the failure of physicians to recommend vaccination to those at risk is consistently cited as one of the biggest barriers to improved uptake.

In a previous survey in our center, we found that in RD patients, from those who had never been vaccinated against influenza (20.2\%), $26.7 \%$ of patients did not consider the influenza vaccine safe and effective vs. $13.5 \%$ among patients who had been vaccinated; only $7.6 \%$ considered that RD patients could not be vaccinated; $11.7 \%$ thought that the influenza vaccine would worsen their RD symptoms; $30.9 \%$ considered other options better than the influenza vaccine; $42.2 \%$ thought that the influenza vaccine weakens the immune system and makes them susceptible to other infections, 33.3\% thought that instead of helping them it would make them worse [16].

We present the preliminary results of a pilot QI vaccination program in RD patients after a year of implementation.

Some strengths are worth mentioning, including: first, the cost-free nature of the program; second, it is a real-world running and evolving $\mathrm{Q}$ program driven by rheumatology fellows-in-training, which results in better development for the future rheumatologist and at the same time it improves the patient care; and third, the inclusion of a multidimensional approach (patients, physicians, and health system).

\section{Study limitations}

Our study is not without limitations. Among them are: first, the self-reported nature of the questionnaires; second, the lack of sample size calculation in our method and the implicit limitations of the sampling technique used in this study, which is reflected in our inability to detect a statistically significant change in vaccination uptake; third, the short period of evaluation considering the seasonal trend of vaccination and the fact that some of the vaccines evaluated have a longer interval between recommended times for application.

\section{Conclusions}

The implementation of a pilot QI program coordinated by rheumatology fellows-in-training showed promising benefits in the vaccination uptake among RD patients. Quality improvement interventions may be helpful to improve vaccination rates in this complex population and help to evaluate the barriers to surpass.

\section{Acknowledgments}

To the nurses and administrative personnel of our clinic. Preliminary results of this study (as abstract AB1158) were accepted for publication in the EULAR 2020 E-congress and presented as a poster in the PANLAR 2020 Virtual Congress (abstract 166).

The authors declare no conflict of interest.

\section{References}

1. Furer V, Rondaan C, Heijstek M, et al. Incidence and prevalence of vaccine preventable infections in adult patients with autoimmune inflammatory rheumatic diseases (AIIRD): a systemic literature review informing the 2019 update of the EULAR recommendations for vaccination in adult patients with AIIRD. RMD Open 2019; 5: e001041, DOI: 10.1136/rmdopen-2019-001041.

2. Papp KA, Haraoui B, Kumar D, et al. Vaccination guidelines for patients with immune-mediated disorders taking immunosuppressive therapies: executive summary. J Rheumatol 2019; 46: 751-754, DOI: 10.3899/jrheum.180784.

3. Dubé E, Laberge C, Guay M, et al. Vaccine hesitancy: an overview. Hum Vaccin Immunother 2013; 9: 1763-1773, DOI: 10.4161/hv.24657.

4. Furer V, Rondaan C, Heijstek MW, et al. 2019 update of EULAR recommendations for vaccination in adult patients with autoimmune inflammatory rheumatic diseases. Ann Rheum Dis 2020; 79: 39-52, DOI: 10.1136/annrheumdis-2019-215882.

5. Singh JA, Saag KG, Bridges SL Jr, et al. 2015 American College of Rheumatology Guideline for the treatment of rheumatoid arthritis. Arthritis Rheumatol 2016; 68: 1-26, DOI: 10.1002/ art.39480.

6. Rubin LG, Levin MJ, Ljungman P, et al. 2013 IDSA clinical practice guideline for vaccination of the immunocompromised host. Clin Infect Dis 2014; 58: 309-318, DOI: 10.1093/cid/cit816.

7. Ledingham J, Gullick N, Irving K, et al. BSR and BHPR guideline for the prescription and monitoring of non-biologic disease-modifying anti-rheumatic drugs. Rheumatology (Oxford) 2017; 56: 2257, DOI: 10.1093/rheumatology/kex389.

8. Cardiel MH, Carrillo S, Pérez M, et al. Update of the Mexican College of Rheumatology guidelines for the pharmacological treatment of rheumatoid arthritis, 2018. Reumatol Clin 2021; 17: 215-228, DOI: 10.1016/j.reumae.2019.04.001 [Article in Spanish].

9. Desai SP, Lu B, Szent-Gyorgyi LE, et al. Increasing pneumococcal vaccination for immunosuppressed patients: a cluster 
quality improvement trial. Arthritis Rheum 2013; 65: 39-47, DOI: 10.1002/art.37716.

10. Baker DW, Brown T, Lee JY, et al. A multifaceted intervention to improve influenza, pneumococcal, and herpes zoster vaccination among patients with rheumatoid arthritis. J Rheumatol 2016; 43: 1030-1037, DOI: 10.3899/jrheum.150984.

11. Harrison N, Poeppl W, Miksch M, et al. Predictors for influenza vaccine acceptance among patients with inflammatory rheumatic diseases. Vaccine 2018; 36: 4875-4879, DOI: 10.1016/j. vaccine.2018.06.065

12. Hmamouchi I, Winthrop K, Launay O, Dougados M. Low rate of influenza and pneumococcal vaccine coverage in rheumatoid arthritis: data from the international COMORA cohort. Vaccine 2015; 33: 1446-1452, DOI: 10.1016/j.vaccine.2015.01.065.

13. Brocq O, Acquacalda E, Berthier F, et al. Influenza and pneumococcal vaccine coverage in 584 patients taking biological therapy for chronic inflammatory joint: a retrospective study. Joint Bone Spine 2016; 83: 155-159, DOI: 10.1016/j.jbspin. 2015.11.005.

14. Murray K, O'Rourke A, Low C, et al. AB1250 Vaccination awareness and uptake in inflammatory arthritis patients. Ann Rheum Dis 2018; 77 (Suppl 2): 1721, DOI: 10.1136/annrheumdis-2018-eular.2231.

15. Jiang Y, Zhang X, Lv Q, et al. Knowledge, attitude, and practice regarding infection and vaccination in patients with rheumatic diseases in China. Hum Vaccin Immunother 2019; 15: 1100-1105, DOI: 10.1080/21645515.2019.1568160.

16. Figueroa-Parra G, Esquivel-Valerio JA, Santoyo-Fexas L, et al. Knowledge and attitudes about influenza vaccination in rheumatic diseases patients. Hum Vaccin Immunother 2021; 17: 1420-1425, DOI: 10.1080/21645515.2020.1816108. 